\title{
Nonlinear Optical Properties of Novel Polypyrrole Derivatives Bearing Different Aromatic Segments
}

\author{
Wei Zhao $^{1^{*}}$, Yun Wang2, Aijian Wang2* \\ ${ }^{1}$ School of Energy \& Power Engineering, Jiangsu University, Zhenjiang, China \\ ${ }^{2}$ School of Chemistry \& Chemical Engineering, Jiangsu University, Zhenjiang, China \\ Email: *wzhao@ujs.edu.cn, ${ }^{\star}$ wajujs@ujs.edu.cn
}

How to cite this paper: Zhao, W., Wang, Y. and Wang A.J. (2017) Nonlinear Optical Properties of Novel Polypyrrole Derivatives Bearing Different Aromatic Segments. Materials Sciences and Applications, 8, 774-783. https://doi.org/10.4236/msa.2017.811056

Received: September 22, 2017

Accepted: October 20, 2017

Published: October 23, 2017

Copyright $\odot 2017$ by authors and Scientific Research Publishing Inc. This work is licensed under the Creative Commons Attribution International License (CC BY 4.0).

http://creativecommons.org/licenses/by/4.0/ (c) (i) Open Access

\begin{abstract}
Polypyrrole (PPy) and its derivatives containing different aromatic segments were synthesized by the interfacial polymerization reaction using ammonium per sulfate as oxidant, and were characterized by using Fourier transform infrared, Ultraviolet/visible absorption, and Raman spectroscopic techniques. The third-order nonlinear optical properties of all samples were measured by using nanosecond Z-scan measurements at $532 \mathrm{~nm}$. At the identical mass concentration of $0.15 \mathrm{mg} \cdot \mathrm{mL}^{-1}$, all PPy derivatives exhibited an obvious reverse saturable absorption performance, while the saturable absorption response was observed for PPy. In addition, significant differences in their nonlinear optical performances were observed, highlighting the influence on optical nonlinearity of the aromatic segments of conjugated polymers.
\end{abstract}

\section{Keywords}

Polypyrrole, Conjugated Polymers, Nonlinear Optical, Aromatic Segments

\section{Introduction}

Nonlinear optical (NLO) materials were investigated quite intensively in recent years due to their widespread applications in information storage, optical communication, optical switching, optical limiting, optical computing, logic devices, and image transmission [1] [2]. During past decades, organic materials have attracted great attention as NLO materials owing to their potential advantages over the classical inorganic materials in many aspects such as higher laser damage threshold, and flexible tailorability [3] [4]. Among these organic materials, conjugated polymers with largely delocalized $\pi$-electrons on its main chain are 
emerging as most widely studied materials in the research area of NLO, due to their superiority in favourable chemical stability, easy processability, good structural and mechanical properties, which have made them as active materials in devices fabrication [5] [6] [7]. It is of vital important to establish the inherent relationship between the NLO properties and chemical structure of conjugated polymers.

Recently, polypyrrole ( $\mathrm{PPy}$ ) has been regarded as a typical representative of conducting polymer for electronic applications [8]. In addition, PPy and its derivatives are promising materials for rechargeable batteries, high-capacity electrode, biosensors and immune sensors for its excellent properties, such as high conductivity, controllable chemical and electrochemical properties, good environmental stability and easy processability [9] [10]. Generally, PPy can be easily prepared by chemical and electrochemical methods. Nevertheless, to the best of our knowledge, there are few investigations on NLO properties of PPy and its derivatives.

A large amount of studies indicated that the substituent groups of the molecule have intimate influence on its NLO properties [11]. Herein, with the goal to investigate the relationship between structure and NLO properties, we have synthesized PPy and five PPy derivatives by chemical oxidation method. By using various aldehydes which possess different substituent groups as the reactant to prepare the PPy derivatives, we studied the influence of different substituent groups on its structure and NLO properties. A comprehensive study and characterization of the PPy derivatives by using FTIR, UV/vis, and Raman analysis are reported in this work. The Z-scan technique in the nanosecond regime at 532 nm was used to study the NLO properties of PPy and its derivatives with different substituent groups. The as-prepared PPy and its derivatives (PPy-1, PPy-2, PPy-3, PPy-4, and PPy-5) can be used as NLO materials and the research may be useful to rational design and prepare the NLO materials.

\section{Experimental Section}

\subsection{Materials and Reagents}

Pyrrole monomer, benzaldehyde, and 4-methylbenzaldehyde (Shanghai Sinopharm Chemical Reagent Co. Ltd, China) were distilled under reduced pressure before use. Ammonium persulfate (APS, Shanghai Sinopharm Chemical Reagent Co. Ltd, China) as an oxidant was analytical grade and used as received. 4-Bromobenzaldehyde, 4-hydroxybenzaldehyde, 4-N,N-diethylamino-benzaldehyde, and other reagents were purchased from commercial suppliers and used without further purification. Deionized water was used throughout the whole sample preparation process.

\subsection{Preparation of PPy and Its Derivatives}

The typical chemical redox oxidative polymerization method for preparation of the PPy and its derivatives was carried out as follows (Scheme 1): In a $50 \mathrm{~mL}$ 

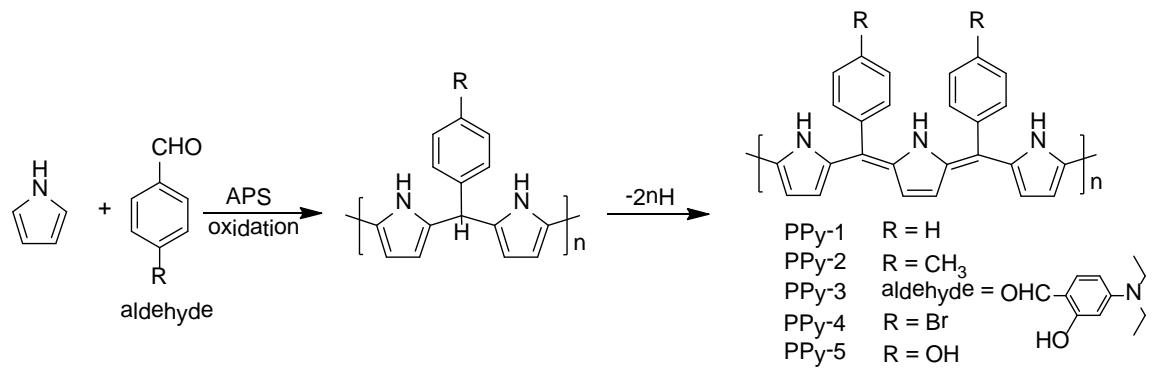

Scheme 1. Synthetic routes toward new PPy polymers.

single-neck round-bottomed flask, $0.2 \mathrm{~mL}(0.9 \mathrm{mmoL})$ of pyrrole and $1.5 \mathrm{mmo}-$ Laldehydes were dissolved in $10 \mathrm{~mL}$ dichloromethane, the mixture was stirred for $30 \mathrm{~min}$, and then $0.2 \mathrm{~g}$ APS in $5 \mathrm{~mL}$ deionized water was added slowly by a constant pressure drop funnel accompany with visible liquid-liquid interface. The resultant mixture was magnetically stirred at room temperature. The solution near the interface turned dark quickly, indicating that the start of the polymerization. The reaction was kept at room temperature for 12 hours. Black precipitate was filtered and washed several times with deionized water, methanol, and ethanol. The product was dried under vacuum at room temperature for 2 days. These PPy derivatives are denoted aspoly\{(pyrrole-2,5-diyl) [ben- zylidene]\} (PPy-1), poly\{(pyrrole-2,5-diyl)[4-methylbenzylidene]\}(PPy-2), poly $\{$ (pyrrole-2,5-diyl) [(4-N,N-diethylamino-2-hydroxy)benzylidene $\}$ (PPy-3), poly $\{($ pyrrole-2,5-diyl)[4-bromobenzylidene] $\}(\mathrm{PPy}-4)$, and $\operatorname{poly}\{($ pyrrole-2,5-diyl) [4-hydroxybenzylidene]\} (PPy-5). The method to synthesis PPy is the same as those PPy derivatives in the absence of the aldehydes.

\subsection{Instruments and Measurements}

Fourier transform infrared (FTIR) spectra were measured with a MB154S-FTIR system (Canada) using spectroscopic grade $\mathrm{KBr}$ pellet. Raman spectra were carried out at room temperature on a Renishaw In via Raman Microscope. The 532 $\mathrm{nm}$ line of an $\mathrm{Ar}^{+}$radiation was used as the excitation resource, which was focused onto samples by using a microscope equipped with a $\times 100$ objective. The ultraviolet/visible (UV/Vis) spectra were obtained by a JASCO V-570 spectrophotometer using $1 \mathrm{~cm}$ quartz cuvette. Elemental analyses were obtained on a Perkin-Elmer 240C CHN elemental analyzer.

The nonlinear optical performances of all samples, including PPy, PPy-1, PPy-2, PPy-3, PPy-4, and PPy-5, were assessed by the Z-scan measurement with linearly polarized $4 \mathrm{~ns}$ laser pulses at $532 \mathrm{~nm}$ with a repetition rate of $2 \mathrm{~Hz}$. DMF suspension solutions of all samples in quartz cells of $2 \mathrm{~mm}$ thickness were placed at the focal point of a lens with a focal length of $50 \mathrm{~cm}$, which was controlled by a computer, and then moved toward the z-axis of the incident beam. The incident and transmitted pulse intensity were measured by two energy detectors (Rjp-765 energy probe), which were linked to an energy meter (Rj-7620 ENERGY RATIOMETER, Laserprobe). 


\section{Results and Discussion}

\subsection{FTIR Studies}

Fourier transform infrared spectroscopy (FTIR) has been used to study the structure of PPy and its derivatives. In Figure 1, FTIR spectra of PPy and its derivatives in the range $4000-400 \mathrm{~cm}^{-1}$ were given to illustrate the chemical environment of the samples. For PPy, the characteristic broad band at $3425 \mathrm{~cm}^{-1}$ corresponds to the $\mathrm{N}-\mathrm{H}$ stretching vibration of the pyrrole rings, and may also be ascribed to the $\mathrm{O}-\mathrm{H}$ stretching vibration of water from the moisture in the air [12]. The peak at $1704 \mathrm{~cm}^{-1}$ is ascribed the over oxidation of the pyrrole in the process of fabricating PPy. The two peaks observed at 1562 and $1476 \mathrm{~cm}^{-1}$ are attributed to the $\mathrm{C}=\mathrm{C}$ and $\mathrm{C}-\mathrm{N}$ stretching vibrations, respectively. The peak at $1299 \mathrm{~cm}^{-1}$ is ascribed to the C-C in-ring stretching and C-N in-plane deformation modes. The peaks observed at 1208 and $1050 \mathrm{~cm}^{-1}$ correspond to $\mathrm{C}-\mathrm{H}$ in-plane vibration and $\mathrm{C}-\mathrm{N}$ stretching vibration [13], respectively. The band at $925 \mathrm{~cm}^{-1}$ is due to C-H out-of-plane deformation, and the peak observed at 800 $\mathrm{cm}^{-1}$ is associated with C-H out-of-plane vibration [14]. The band at $680 \mathrm{~cm}^{-1}$ is attributed to the $\mathrm{C}-\mathrm{C}$ out-of-plane ring deformation or $\mathrm{C}-\mathrm{H}$ rocking vibration [15]. As to PPy-1, PPy-2, PPy-3, and PPy-4, all of them show the corresponding characteristic peaks of PPy in the FTIR spectra at around 1549, 1473, 1283, 1193, 1041, 911, and $790 \mathrm{~cm}^{-1}$, respectively. However, those bands are accompanied with obvious bathochromic-shift compared to PPy, ascribing to the influence of the substituent groups in the PPy derivatives. Remarkably, the spectrum of PPy-5 exhibits several bands at 1675, 1596, 1243, 1170, 1041, and $765 \mathrm{~cm}^{-1}$, which can be owed to the influence of its $-\mathrm{OH}$ substituent groups.

\subsection{Linear Optical Absorption Analysis}

$\mathrm{UV} /$ vis spectroscopy is taken to illustrate the inner effect of different substituent groups in those PPy derivatives on the photophysical properties. UV/vis spectra

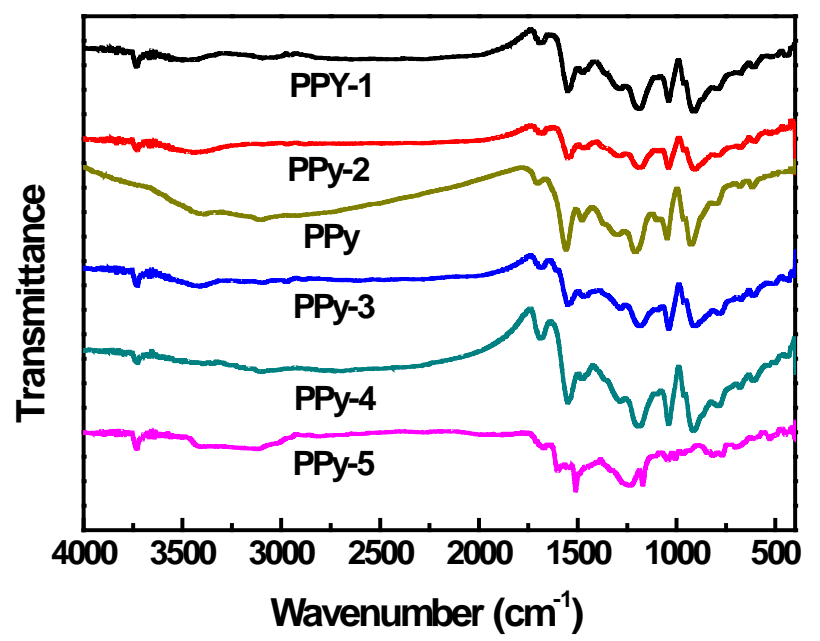

Figure 1. FTIR spectra of PPy and its derivatives (PPy-1, PPy-2, PPy-3, PPy-4 and PPy-5). 
of PPy and its derivatives (PPy-1, PPy-2, PPy-3, PPy-4 and PPy-5) in DMF are presented in Figure 2. In the PPy spectrum, it shows a strong peak at $280 \mathrm{~nm}$ and a broad absorption band in the range $400-650 \mathrm{~nm}$. The former is contributed to the $\pi-\pi^{*}$ interband transition, namely, transition from valence band to the conduction band of neutral state of PPy, which is indicative of the formation of PPy [10]. As for the broad absorption band, it reveals the take place of the polymerization [16]. For those PPy derivatives, it is obvious to find that all of them have absorption peak at around $280 \mathrm{~nm}$, but with some shift in the peak position. In particular, PPy-3 shows three absorption bands at 268, 305, and $500 \mathrm{~nm}$, while PPy and other PPy derivatives only possess two absorption bands. In addition, compare to PPy and other PPy derivatives, the first peak shifts from 280 to $268 \mathrm{~nm}$. As is known to all, the order of electron-donating ability for these substituent groups is $-\mathrm{N}\left(\mathrm{CH}_{2} \mathrm{CH}_{3}\right)_{2}>-\mathrm{OH}>-\mathrm{CH}_{3}>-\mathrm{H}>-\mathrm{Br}$. Therefore, it is reasonable to deduce that the strong electron-donating ability results in the difference of PPy-3 in comparison with those of others. It is also easy to find that the spectrum of PPy-5 is clearly different from other compounds for the appearance of the second absorption band at $423 \mathrm{~nm}$. But PPy-1 and PPy-2 only show a broad absorption band in the range 400 - 550 and $400-650 \mathrm{~nm}$, respectively. Moreover, PPy-4 exhibited two absorption peaks at 320 and $562 \mathrm{~nm}$. These differences for PPy and its derivatives in the UV/vis spectra should be ascribed to the effect of the diverse substituent groups.

\subsection{Raman Spectra Analysis}

Raman spectroscopy was taken as an effective tool to characterize the structure of the synthesized samples. The Raman spectra of PPy and its derivatives were shown in Figure 3. For the spectrum of PPy, it exhibits two characteristic peaks at 1353 ( $\mathrm{D}$ band) and $1571 \mathrm{~cm}^{-1}$ ( $\mathrm{G}$ band) that correspond to the $\mathrm{C}=\mathrm{C}$ backbone

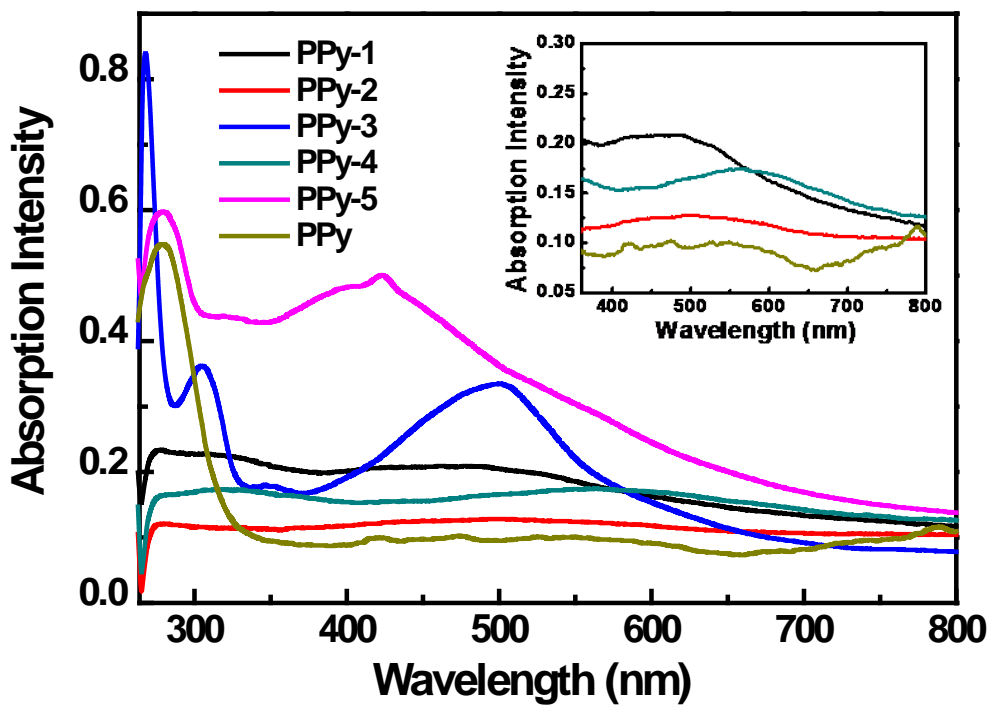

Figure 2. UV/vis spectra of PPy and its derivatives (PPy-1, PРy-2, PPy-3, PPy-4 and PPy-5). 


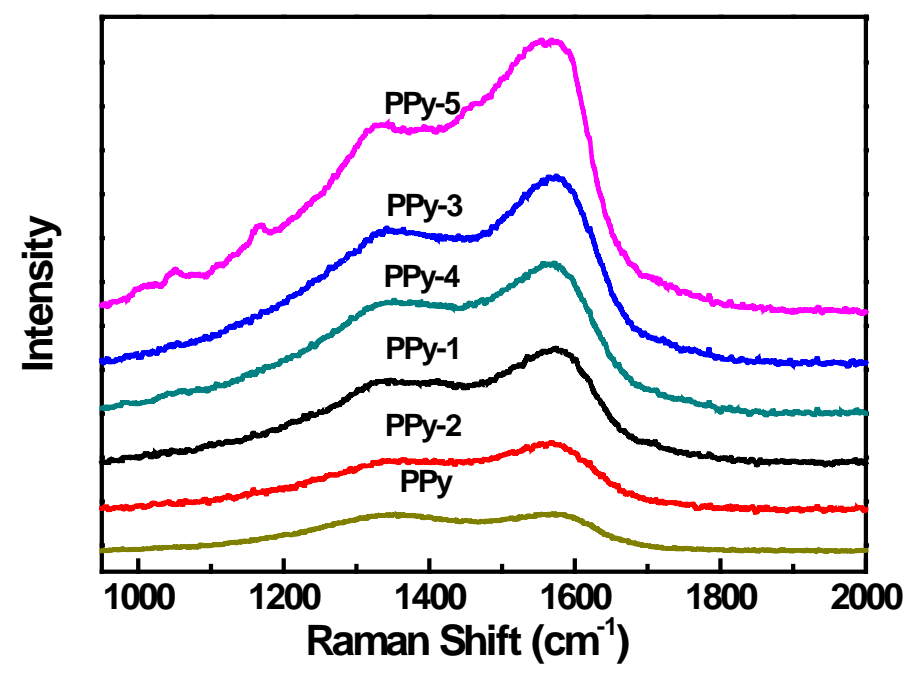

Figure 3. Raman spectra of PPy and its derivatives (PPy-1, PPy-2, PPy-3, PPy-4 and PPy-5).

stretching and the ring stretching mode of PPy, respectively [17]. The position and intensity ratio of D and G bands for PPy and its derivatives were shown in Table 1. It is obvious to find that the D and G bands for those PPy derivatives have been shifted for several wave numbers to some extent as compared to PPy. In addition, PPy-1, PPy-2, PPy-3, PPy-4 and PPy-5 also show distinction to each other, which indicate the significant effect of varying substituent groups on the Raman data. Similar to graphene, the intensity ratio of $D$ and $G$ bands $\left(I_{D} / I_{G}\right)$ was used to investigate the extent of defects in PPy and its derivatives [18]. From Table 1, we can find that the $I_{D} / I_{G}$ of PPy derivatives decreased largely from 0.98 to $\sim 0.69$, indicating that PPy derivatives have less disorder structure and defects than PPy.

\subsection{Nonlinear Optical Performances}

The NLO properties of conjugated polymers are important for applications such as optical limiting, optical processing and optical switching [19]. In the last few years, process in the field of photonics and optoelectronics has deeply promoted the search for materials with excellent NLO properties and ultrafast response, with the goal of protecting sensitive optical systems and human eyes [20]. As a consequence, these properties have been widely investigated using several experimental techniques over the past years. The Z-scan technique is a sensitive method to characterize the NLO properties of materials with a simple experimental setup. In the Z-scan technique, the sample moves along the beam propagating direction (Z-axis) through the focus of a lens, and as it moves the sample is under variable irradiance. The total transmittance through the sample is recorded as a function of incident laser intensity at each z-position and analyzed using Z-scan theory.

Conjugated polymersexhibit excellent NLO activities because of their extensive $\pi$ electron delocalization and macrocyclic conjugate structure [10], so it was 
Table 1. The position and intensity raito of D and G bands for PPy and its derivatives (PPy-1, PPy-2, PPy-3, PPy-4 and PPy-5).

\begin{tabular}{cccc}
\hline Compounds & $\begin{array}{r}\text { D band Position } \\
\left(\mathrm{cm}^{-1}\right)\end{array}$ & $\begin{array}{c}\text { G band Position } \\
\left(\mathrm{cm}^{-1}\right)\end{array}$ & $\begin{array}{c}\text { Intensity ratio } \\
\left(\mathrm{I}_{\mathrm{D}} / \mathrm{I}_{\mathrm{G}}\right)\end{array}$ \\
\hline PPy & 1353 & 1571 & 0.98 \\
PPy-1 & 1347 & 1574 & 0.71 \\
PPy-2 & 1362 & 1569 & 0.73 \\
PPy-3 & 1341 & 1575 & 0.72 \\
PPy-4 & 1352 & 1572 & 0.73 \\
PPy-5 & 1348 & 1568 & 0.69 \\
\hline
\end{tabular}

of tremendous interests to evaluate the optical nonlinearities of the PPy and its derivatives. The samples were prepared by dissolving $1.5 \mathrm{mg}$ of PPy, PPy-1, PPy-2, PPy-3, PPy-4, and PPy-5 into $10 \mathrm{~mL}$ of DMSO, respectively, followed with 30 min of ultrasonic treatment.

The open-aperture Z-scan results of the tested samples at $532 \mathrm{~nm}$ are presented in Figure 4. Because the solvent DMSO exhibits no nonlinear absorption under these experimental conditions, the observed nonlinear absorption must arise from the solutes. At the excitation wavelength, the pristine PPy exhibits clear saturable absorption process; that is, the total transmission increases as the sample moves to the higher irradiance at the focal point [10]. In contrast, valley-shaped curves indicate the occurrence of nonlinear absorption and optical limiting behaviors. The depth of the valley in the Z-scan curves correlates with the extent of optical limiting [21] [22]. The decrease in normalized transmittance of PPy-3 with increasing fluence exceeds that all of other samples in the present study. The order of the decreases in normalized transmittance (PPy-3 > PPy-5 $>$ PPy-2 $>$ PPy-1 > PPy-4) indicate that the optical limiting response of PPy-3 is superior to those of the other samples, highlighting the influence on optical nonlinearity of the composition and aromatic segments of conjugated polymers. The effective NLO performance makes $\mathrm{PPy}-3$ possible candidates for practical applications as mode-locking elements or optical limiters in eyes and sensors protection for ns pulses. Although more work is needed to determine other potential applications of the novel systems, this approach opens new possibilities for research on the construction of conjugated polymers, and it may be extended to other nanostructured materials in which agglomeration is not expected.

\section{Conclusion}

In summary, we have reported the synthesis of PPy and its derivatives containing different aromatic segments by the interfacial polymerization reaction. The structural properties of all samples were characterized by using FTIR, UV/vis, and Raman techniques. Their NLO properties have been investigated using the Z-scan technique at $532 \mathrm{~nm}$ with $4 \mathrm{~ns}$ laser pulses. PPy-3 exhibited increased 


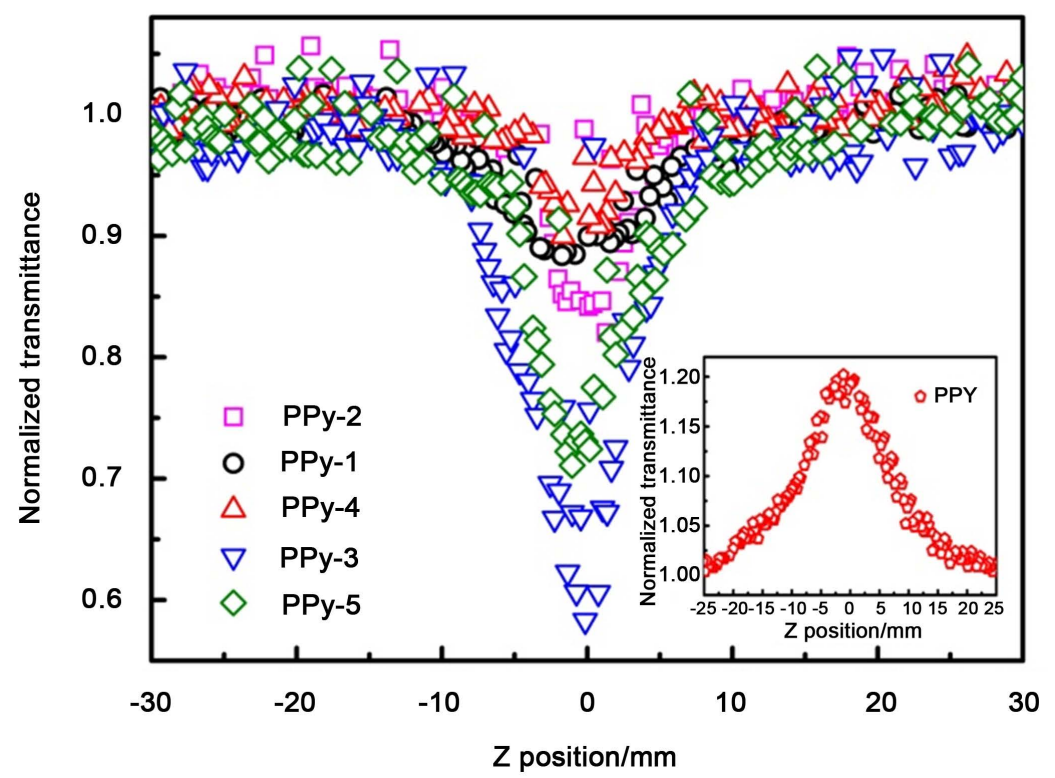

Figure 4. Normalized open-aperture Z-scan data of PPy and its derivatives (PPy-1, PPy-2, PPy-3, PPy-4 and PPy-5) at a wavelength of $532 \mathrm{~nm}$ in the ns regime.

NLO properties in the nanosecond regime. The aromatic segments are found to be an important factor influencing the NLO performances of the PPy derivatives. The present work revealed important insights into the NLO responses of conjugated polymers, and can provide new guidelines for the design of polymers-based NLO materials.

\section{Acknowledgements}

This research was supported financially by the National Natural Science Foundation of China (51506077), the Natural Science Foundation of Jiangsu Province (BK20150488), the Natural Science Foundation of Jiangsu High School (15KJB430007, 15KJB610003, 15KJD150002), the China Postdoctoral Foundation (2016M601733) and Research Foundation of Jiangsu University (13JDG066, 15JDG156).

\section{References}

[1] Zhu, J., Li, Y., Chen, Y., Wang, J., Zhang, B., Zhang, J. and Blau, W.J. (2011) Graphene Oxide Covalently Functionalized with Zinc Phthalocyanine for Broadband Optical Limiting. Carbon, 49, 1900-1905. https://doi.org/10.1016/j.carbon.2011.01.014

[2] Jiang, L., Jiu, Y., Li, Y.L., Li, Y., Yang, J., Li, J., Li, C., Liu, H. and Song, Y. (2008) Excited-State Absorption and Sign Tuning of Nonlinear Refraction in Porphyrin Derivatives. Journal of Physical Chemistry B, 112, 756-759. https://doi.org/10.1021/jp076515v

[3] Hanack, M., Schneider, T., Barthel, M., Shirk, J.S., Flom, S.R. and Pong, R.G.S. (2001) Indium Phthalocyanines and Naphthalocyanines for Optical Limiting. Coordination Chemistry Reviews, 219, 235-258. https://doi.org/10.1016/S0010-8545(01)00327-7 
[4] Li, G.H., Wang, A.J., Song, J.B. and Zhou, Q. (2015) A Novel Zinc Tetraphenylporphyrinate Substituted in the Axial Position with One E-Stilbazole: Synthesis, Structure, and Nonlinear Optics. Inorganic Chemistry Communication, 57, 47-50. https://doi.org/10.1016/j.inoche.2015.05.002

[5] Osterbacka, R., An, C.P., Jiang, X.M. and Vardeny, Z.V. (2000) Two-Dimensional Electronic Excitations in Self-Assembled Conjugated Polymer Nanocrystals. Science, 287, 839-842. https://doi.org/10.1126/science.287.5454.839

[6] Nalwa, H.S. (1993) Organic Materials for Third-Order Nonlinear Optics. Advanced Materials, 5, 341-358. https://doi.org/10.1002/adma.19930050504

[7] Remyamol, T., John, H. and Gopinath, P. (2013) Synthesis and Nonlinear Optical Properties of Reduced Graphene Oxide Covalently Functionalized with Polyaniline. Carbon, 59, 308-314. https://doi.org/10.1016/j.carbon.2013.03.023

[8] Luo, S., Liu, X., Wu, D., Shi, G. and Mei, T. (2014) Tunable Conversion from Saturable Absorption to Reverse Saturable Absorption in Poly (Pyrrole Methine) Derivatives. Journal of Physical Chemistry C, 2, 8850-8853.

[9] Zhou, C., Zhang, Y., Li Y. and Liu, J. (2013) Construction of High-Capacitance 3D CoO@polypyrrole Nanowire Array Electrode for Aqueous Asymmetric Supercapacitor. Nano Letters, 13, 2078-2085. https://doi.org/10.1021/nl400378j

[10] Wang, A.J., Zhao, W. and Yu, W. (2015) Effect of Acid/Base on the Third-Order Optical Nonlinearity of Polypyrrole. Journal of Molecular Structure, 1099, 291-296. https://doi.org/10.1016/j.molstruc.2015.06.073

[11] De la Torre, G., Vázquez, P., Agulló-López, F. and Torres, T. (2004) Role of Structural Factor in the Nonlinear Optical Properties of Phthalocyanines and Related Compounds. Chemistry Reviews, 104, 3723-3750. https://doi.org/10.1021/cr030206t

[12] Li, X.G., Hou, Z.Z., Huang, M.R. and Moloney, M.G. (2009) Efficient Synthesis of Intrinsically Conducting Polypyrrole Nanoparticles Containg Hydroxyl Sulfoaniline as Key Self-Stabilized Units. Journal of Physical Chemistry C, 113, 21586-21595. https://doi.org/10.1021/jp9081504

[13] He, C., Yang, C. and Li, Y. (2003) Chemical Synthesis of Coral-Like Nanowires and Nanowire Network of Conducting Polypyrrole. Synthetic Metals, 139, 539-545.

[14] Mcgehee, M.D. and Heeger, A.J. (2000) Semiconducting (Conjugated) Polymers as Materials for Solid-State Lasers. Advanced Materials, 12, 1655-1668. https://doi.org/10.1002/1521-4095(200011)12:22<1655::AID-ADMA1655>3.0.CO;22

[15] Blinova, N.V., Stejskala, J., Prokes, J. and Omastova, M. (2007) Polyaniline and Polypyrrole: A Comparative Study of the Preparation. European Polymer Journal, 43, 2331-2341.

[16] Konwer, S., Maiti, J. and Dolui, S.K. (2011) Preparation and Optical/Electrical/Electrochemical Properties of Expanded Graphite-Filled Polypyrrole Nanocomposite. Material Chemistry \& Physics, 128, 283-290.

[17] Xie, Y. and Du, H. (2015) Electrochemical Capacitance of Carbon Quantum Dots-Polypyrrole/Titania Nanotube Hybrid. RSC Advances, 5, 89689-89697. https://doi.org/10.1039/C5RA16538E

[18] Singh, A. and Chandra, A. (2013) Graphite Oxide/Polypyrrole Composite Electrodes for Achieving High Energy Density Supercapacitors. Journal of Applied Electrochemistry, 43, 773-782. https://doi.org/10.1007/s10800-013-0573-y

[19] Samoc, M., Gauthier, N., Cifuentes, M.P., Paul, F. and Lapinte, C. (2006) Electrochemical Switching of the Cubic Nonlinear Optical Properties of an Aryldiethy- 
nyl-Linked Heterobimetallic Complex between Three Distinct States. Angewandte Chemie International Edition, 45, 7376-7379.

https://doi.org/10.1002/anie.200602684

[20] He, G.S., Tan, L.S., Zheng, Q. and Prasad, P.N. (2008) Multi-Photon Absorbing Materials: Molecular Designs, Syntheses, Characterizations, and Applications. Chemistry Reviews, 108, 1245-1330. https://doi.org/10.1021/cr050054x

[21] Zhao, W., Wang, Y., Wang, A.J., Qian, J., Zhu, W.H., Dou, S.P., Wang, Q., Zhong, Q. and Chen, A.H. (2017) Novel $\mathrm{Bi}_{2} \mathrm{O}_{2} \mathrm{CO}_{3} /$ Polypyrrole/g- $\mathrm{C}_{3} \mathrm{~N}_{4}$ Nanocomposites with Photocatalytic and Nonlinear Optical Properties. RSC Advances, 7, 7658-7670. https://doi.org/10.1039/C6RA28346B

[22] Martin, R.B., Li, H., Gu, L., Kumar, S., Sanders, C.M. and Sun, Y. (2005) Superior Optical Limiting Performance of Simple Metalloporphyrin Derivatives. Optical Materials, 27, 1340-1345. 